\title{
Effects of Continuous Theta Burst Stimulation Over the Left Dlpfc on Mother Tongue and Second Language Production In Late Bilinguals: A Behavioral and ERP Study
}

\author{
Lea B. Jost ${ }^{1}$ D $\cdot$ Maria I. Pestalozzi ${ }^{1} \cdot$ Dario Cazzoli ${ }^{2,3} \cdot$ Michael Mouthon $^{1} \cdot$ René M. Müri $^{2,3} \cdot$ Jean-Marie Annoni $^{1}$
}

\begin{abstract}
Clinical, neuroimaging, and non-invasive brain stimulation studies have associated the dorsolateral prefrontal cortex (DLPFC) with the multilingual language control system. Here, we investigated if this role is increased during the processing of the non-dominant language due to the higher cognitive/attentional demands. We used an inhibitory repetitive transcranial magnetic stimulation (rTMS) protocol over the left DLPFC and investigated the behavioral and electrophysiological effects on (i) picture naming in the mother tongue and second language, (ii) forward and backward translation and (iii) non-verbal inhibition. To this end, we compared the effects of inhibitory rTMS (cTBS) vS sham-rTMS using a single-blind within-subject design including 22 late bilinguals. Behaviorally, response times were longer after cTBS compared to sham-rTMS in the picture naming task independent of language, while response times were not affected for the word translation task. These results were mirrored on the electrophysiological level showing an effect of stimulation in the picture naming task starting at $547 \mathrm{~ms}$ post-stimulus onset, but not in the translation task. This late time range is likely associated with processes of conflict resolution and initiation of the articulation of the word rather than processes related to lexical selection or language switching. For the non-verbal inhibition task, behavioral outcome was not affected despite electrophysiological stimulation-induced changes. Overall, the results suggest that the DLPFC plays a role in top-down cognitive control in language production, but that this role is not increased with higher cognitive demand such as naming in a second language or in language switching during word translation.
\end{abstract}

\section{Introduction}

Clinical and neuroimaging studies indicate that language processing relies both on a dedicated language network and on a domain-general cognitive control network, especially

Handling Editor: Gregor Thut

Lea B. Jost

lea.jost@unifr.ch

1 Laboratory for Cognitive and Neurological Sciences, Neurology Unit, Department of Neuroscience and Movement Science, Faculty of Science and Medicine, University of Fribourg, Ch. du Musée 5, CH-1700 Fribourg, Switzerland

2 Gerontechnology and Rehabilitation Group, ARTORG Center for Biomedical Engineering Research, University of Bern, Bern, Switzerland

3 Perception and Eye Movement Laboratory, Department of Neurology, University of Bern, Bern, Switzerland when it comes to bilingual language processing (for reviews see Hervais-Adelman et al. 2011; Ye and Zhou 2009).

A large number of models of bilingual word access have been brought forward to explain how words in a target language are selected and how co-activation and thus potential intrusion from an unintended language can be inhibited (Bloem and La Heij 2003; Dijkstra and van Heuven 2002; Green 1998; Kroll and Stewart 1994). Despite some debate about which models explain bilingual word production best, there seems to be converging evidence that the activation of two languages gives rise to high demands on cognitive control (Green and Abutalebi 2013; Kroll et al. 2014; Kroll and Bialystok 2013; Luk et al. 2012; Mouthon et al. 2019). Abutalebi and Green (2008; Green and Abutalebi 2013; 2007) suggest that bilinguals cope with the potential interference by recruiting at least partly domain-general cognitive control regions, such as the anterior cingulate cortex (ACC), the dorsolateral prefrontal cortex (DLPFC), the inferior parietal lobule and the basal ganglia. These brain regions are traditionally associated with domain general executive 
control implicated in diverse processes such as response selection, task-switching and task-set reconfiguration, prevention of interference, inhibition, planning and working memory (Badre and Wagner 2004; Brunoni and Vanderhasselt 2014; Hart et al. 2013; Liston et al. 2006). The widely applied model by Miyake et al. (2000) includes three main components of executive control: (a) inhibition (interference control), which comprises the ability to suppress or lower unwanted dominant or automatic responses; (b) updating, which is closely linked to the notion of working memory, and refers to the ability of monitoring information of relevance to the task at hand and appropriately revise items held in short term memory by replacing no longer relevant information with newer information; (c) shifting, which refers to the ability to switch back and forth between multiple tasks, mental sets, or distinct aspects of a given task or problem. Several theories suggest a functional role of these executive functions in mono- and bilingual word production, as for example the computational model WEAVER ++ (Word Encoding by Activation and VERification, Roelofs 2018; Roelofs and Piai 2011). In this model, planning and selection processes are supported by working memory and sustained attention, which can in turn influence the time-course of the naming process. Working memory processes help to successfully update the selection-subgoals, starting from the selection of the word (lemma), and ending with the selection of the motor program for producing the phonemes (Roelofs and Piai 2011). Sustained attention and monitoring keep the activation of the target linguistic units high, and thus lower their threshold compared to the one of competing activated units. Alternatively, Nozari (2019) concluded that lexical access not only depends on enhanced activation of the target units, but also on inhibition of competing units. Following these models, it can thus be hypothesized that both updating and inhibition contribute to successful word production. A role of such processes has also been suggested in theories of speech production in multilingual contexts (Green 1998), when participants have to keep both languages activated and monitor incoming contextual information in order to use the appropriate language. There might thus be an overlap of executive control functions (i.e. inhibition and working memory) involved in word retrieval in both mono- and bilingual contexts.

According to Roelofs (2008), the control functions inhibition, updating and shifting mainly rely on the anterior cingulate cortex (ACC) and lateral PFC. In particular, a role of the dorsolateral prefrontal cortex (DLPFC) in language control has also been put forward by several neuroimaging (Hernandez et al., 2001, 2000), intracranial electric stimulation (Lubrano et al. 2012; Sierpowska et al. 2018) as well as non-invasive brain stimulation (NIBS) studies. Several studies have shown that the left DLPFC is linked to the executive control of multiple language use, especially during language switching (Holtzheimer et al. 2005; Nardone et al. 2011). However, the involvement of this region in intra-language control is less clear. Despite several tDCS and rTMS studies in healthy subjects and patients indicating a role of the left DLPFC in intra-language tasks, e.g. lexical access (Cappa et al. 2002; Fertonani et al. 2010), verbal fluency (Iyer et al. 2005) and sentence comprehension (Cotelli et al. 2011), the nature of this control process in language production has yet to be resolved. Moreover, it remains unclear whether the involvement of the left DLPFC in bilingualism is higher when producing words in L2, as compared to the more dominant L1. It also remains unresolved when and how the left DLPFC might help in the control of successful retrieval and production of words.

rTMS is a non-invasive brain stimulation method that can induce neuroplasticity depending on the stimulation protocol used. A stimulation protocol that has shown much promise is the so-called theta burst stimulation (TBS) protocol, during which short bursts of high frequency magnetic pulses at $50 \mathrm{~Hz}$, repeated at $5 \mathrm{~Hz}$, are applied (Huang et al. 2005). Depending on the pattern of application, the resulting effects can be either facilitatory (intermittent TBS, iTBS) or suppressive (continuous TBS, cTBS), resulting from modifications in the strength of synaptic connections by mechanisms that are similar to long-term potentiation or depression. The advantages of cTBS over the more traditional rTMS protocol are primarily the shorter stimulation times, the sub-threshold stimulation intensities, and the longer-lasting offline stimulation effects (Goldsworthy et al. 2012).

Another useful method to gain information about the timing, activation and interaction of brain networks are event related potentials (ERPs). Instead of analyzing amplitudes and occurrence of pre-defined ERP components, we chose a global, data-driven ERP analysis approach that allows differentiating topographic and electrical field strength measures (e.g. De Pretto et al. 2017; Jost et al. 2018; Pestalozzi et al. 2020). To this end, time windows during which conditions of interest differ are first identified applying time-point-totime-point topographic analyses of variance (TANOVA). Further investigation of these time windows can then reveal if potential effects are reflected by different source configurations (i.e., reflected in differences in topography), or different activation strength of the same sources (i.e., reflected in differences in global field power, GFP; Michel et al. 2004). This approach thus allows a more physiologically oriented interpretation (Murray et al. 2008) of the underlying cognitive effects of interest.

In the present study, we investigated the effects of an inhibitory cTBS protocol over the left DLPFC on (1) picture naming in L1 (mother tongue) and L2 (second language), (2) word translation and (3) non-verbal inhibition. Based on the assumption that cTBS lowers cortical activity (Rossi et al. 2009), a notion supported by studies showing that cTBS 
leads to profound attenuations of motor evoked potentials (Goldsworthy et al. 2012; Huang et al. 2005) as well as to attenuated cortical oxygenation over the area of stimulation in near-infrared spectroscopy paradigms (e.g. Tupak et al. 2013), we hypothesized to observe slower response times (RTs) after cTBS compared to sham stimulation in all tasks. Due to higher cognitive/attentional demand for forward (L1-L2) compared to backward (L2-L1) translation (Francis and Gallard 2005; Jost et al., 2018b; Kroll and Stewart 1994; Sholl et al. 1995) as well as greater difficulty of picture naming in the nondominant language (Francis et al. 2003; Radman et al. 2018), we moreover expected a stronger inhibitory effect on forward translation and naming in L2. Next to these behavioral outcome measures, we also investigated the effects on the electrophysiological level using ERP data, thus providing the possibility to investigate the neural correlates of the potential behavioral effects.

\section{Materials and Methods}

\section{Participants}

We report data collected from 22 healthy late bilingual adults $(8$ men $)$ aged $18-28$ years $($ mean $=22.4$ years, $\mathrm{SD}=2.2$ years). Their mother tongue was French (L1), and the mean age of acquisition (AoA) of English (L2) was 11.6 $( \pm 1.9)$ years. According to a bilingualism questionnaire and two English proficiency tests (see section "Language evaluation" for details), participants had an intermediate to high L2-proficiency. All subjects were right-handed and had normal or corrected-to-normal vision. None of the participants reported any history of neurological or psychiatric disorders. From an original group of 23, one participant was excluded due to a $30 \mathrm{~s}$ stress-induced vasovagal syncope during the single-pulse motor threshold assessment taking place before the rTMS application. Participants gave written, informed consent and were compensated with $25 \mathrm{CHF} /$ hour for their participation. The study protocol was approved by the local ethics committee and preregistered on "clinicaltrials.gov" (NCT02840396). The experiment was carried out in the Neurology Unit, Department of Medicine, University of Fribourg.

\section{Language Evaluation}

Participants filled out a questionnaire developed by our group (Buetler et al. 2014, 2015 ; Jost et al., 2018) in which they had to indicate the L2-AoA and how present L2 is in their daily activities (i.e. with friends, family, watching TV/ listening to the radio, reading books, and performing mental arithmetics, see Table 1). Participants also self-evaluated their L2-reading, speaking and comprehension skills on a
Table 1 L2-proficiency skills of participants $(n=22)$

\begin{tabular}{llllll}
\hline Variable & mean & SD & & \\
\hline Age of acquisition (years) & 11.59 & 1.64 & & \\
Self-evaluation (\%) & & & & & \\
Speaking & 53 & 13 & & & \\
Comprehension & 71 & 12 & & & \\
Reading & 68 & 14 & & & \\
Writing & 52 & 14 & & & \\
L2 Vocabulary Tests (\% correct) & & & & & \\
DIALANG score & 67 & 14 & & & \\
PVLT high frequency words & 74 & 18 & & & \\
PVLT low frequency words & 38 & 18 & & & \\
PVLT total score & 45 & 13 & & & \\
Immersion & $0 \%$ & $25 \%$ & $50 \%$ & $75 \%$ & $100 \%$ \\
Use of L2 at work/studies (n) & 6 & 12 & 3 & 1 & 0 \\
Consumption of L2-TV/radio (n) & 3 & 11 & 7 & 1 & 0 \\
Use of L2 with friends (n) & 16 & 4 & 2 & 0 & 0 \\
Consumption of L2-books (n) & 9 & 9 & 2 & 1 & 1 \\
\hline
\end{tabular}

$10 \mathrm{~cm}$ visual analogue scale. In addition, each participant performed a sub-test from the computer-based DIALANG language diagnosis system (Zhang and Thompson 2004), in order to obtain an estimation of L2 receptive vocabulary (percentages ranging from 40 to $60 \%$ indicate good basic vocabulary, scores ranging from 60 to $90 \%$ indicate advanced proficiency). Participants also completed the PVLT (Productive Vocabulary Levels Test, Laufer and Nation 1999) to evaluate L2 productive vocabulary. Table 1 provides details on participants' L2-AoA, L2-proficiency skills and their L2-immersion.

\section{Tasks and Procedure}

Participants took part in one cTBS and one Sham-session and were blind with respect to the stimulation condition. Both sessions took place at the same time of the day, with 14 days between the sessions. In each of the two sessions, participants performed a picture naming task, a translation task and a non-verbal Flanker task within the 30mins following the stimulation. The order of tasks as well as the order of stimulation conditions was counterbalanced across participants.

\section{rTMS}

A transcranial magnetic stimulation (TMS) stimulator (MagPro X100, MagVenture) was used to generate repetitive biphasic magnetic pulses. The pulses were delivered with a butterfly-shaped static cooled coil (MCF-B70, MagVenture) with an outer diameter of $97 \mathrm{~mm}$. For the placebo stimulation, we used an MCF-P-B70 coil (MagVenture), which is 
also a butterfly-shaped, static cooled coil with a mechanical outline and sound level identical to the MCF-B70. The coil is equipped with a magnetic shield that provides a field reduction of approximately $80 \%$. We applied a continuous theta burst rTMS (cTBS) protocol (Nyffeler et al. 2006, 2008) with a train of 801 pulses (267 bursts, each burst consisting of three pulses at $30 \mathrm{~Hz}$, repeated at intervals of $100 \mathrm{~ms}$ ). We chose this protocol based on a study comparing the effects of the original cTBS protocol (Huang et al. 2005 ) applied at $80 \%$ of the active motor threshold (AMT), and this modified protocol (Nyffeler et al. 2006) applied at $80 \%$ of the resting motor threshold (RMT) (Goldsworthy et al. 2012). This study revealed that the effects of the modified version on motor evoked potentials are longer lasting and more consistent across subjects. Moreover, this modified stimulation protocol has been used in numerous studies over posterior parietal (e.g. Cazzoli et al. 2015; Cazzoli and Chechlacz 2017; Hopfner et al. 2015; Nyffeler et al. 2009), inferior frontal (e.g. Bohlhalter et al. 2011; Kindler et al. 2012; Vanbellingen et al. 2020), and dorsolateral prefrontal (e.g. Pestalozzi et al. 2020) areas. cTBS was applied over F3 according to the International 10-20 EEG system. This location overlies the left DLPFC (Herwig et al. 2003). The coil was held tangentially to the scalp, with the handle pointing backwards. cTBS was applied at $80 \%$ of subjects' individual resting motor threshold, defined as the minimum stimulator output of TMS single pulses that was able to elicit twitches in the small hand muscles of the right relaxed hand in at least 5 out of 10 trials (two observers). To determine the resting motor threshold, the handle was positioned over the left primary motor cortex, at $45^{\circ}$ with respect to the head's midsagittal plane, the coil's handle pointing backwards. Both the individual motor threshold and the stimulation protocol were applied with the EEG-cap already placed on the head, without electrodes placed at the site of stimulation. The choice of using a stimulation intensity of $80 \%$ RMT (which is larger than what is typically used for studies investigating effects of cTBS over the motor cortex, e.g. Hellriegel et al. 2012; Huang et al. 2005; Steel et al. 2016) was driven by the following: most studies refer to the motor threshold for the individualization of the intensity of stimulation, also when other brain regions are then targeted for stimulation. However, as stated by Rossi et al. (2009) and Lefaucheur et al. (2014), the relationship between the excitability of the motor cortex, visual cortex and other non-motor areas remains to be clarified. Given that stimulation intensity may play a role in the inhibitory effectiveness of cTBS (Goldsworthy et al. 2012), we decided to use an intensity of $80 \%$ RMT to increase the chances of observing an effect of cTBS on brain activity, while at the same time adhering to safety guidelines (Rossi et al. 2009). Importantly, the use of $80 \%$ RMT is also in line with other studies investigating effects of cTBS over the DLPFC (e.g. Bolton and Staines 2011; Lowe et al. 2018;
Pestalozzi et al. 2020; Schicktanz et al. 2015) and a study specifically addressing the safety issue of applying cTBS at $80 \%$ RMT over the DLPFC (Grossheinrich et al. 2009).

After the cTBS and sham sessions, participants rated possible side effects on a scale from 1 (absent) to 4 (severe). No differences were reported for headache, neck pain, burning or prickling sensation on the skin, drowsiness, difficulties in concentrating or change in mood (all p's $>0.05$ ). For reddening of the skin the values were larger after cTBS than Sham $(p=0.046)$, while there was a small trend for more drowsiness after sham compared to cTBS ( $p=0.083$, Wilcoxon signed ranks tests).

\section{Picture naming task}

Participants were asked to overtly name 50 black and white line drawings selected from the database by Cycowicz et al. (1997), which was normed in French by Alario and Ferrand (1999) and in English by Snodgrass and Vanderwart (1980). Following a $200 \mathrm{~ms}$ fixation cross, the pictures were displayed for $2000 \mathrm{~ms}$ and followed by a jittered time interval of 1500-2500 ms. The total response time was 3500$4500 \mathrm{~ms}$ long. The four item lists (session $1 \mathrm{~L} 1-\mathrm{L} 2$, session 1 L2-L1, session 2 L1-L2, session 2 L2-L1) did not contain cognates and were matched for familiarity, AoA ${ }^{1}$ and log-transformed lexical frequency ${ }^{2}$ (Duyck et al. 2004). Moreover, the lists were also matched for name agreement, image agreement and visual complexity, which are major determinants of naming speed (Alario et al. 2004). Note that pictures presented in the English list in the first session were presented in the French list in the second session and vice versa.

E-Prime 2.0 (Psychology Tools, Inc., Pittsburgh, PA, USA) was used for controlling stimulus presentation and response recording. Word productions were systematically checked for accuracy and response latencies (time separating the onset of the picture presented on the screen and the articulation onset) with a speech analysis software (Audacity®) by a person who was blind to the stimulation condition.

\section{Word translation task}

In this overt production task, participants were asked to translate visually presented words either from L1 to L2 (forward translation) or from L2 to L1 (backward translation), respectively. Each of the two subtasks included a total of 50 trials. Following a $200 \mathrm{~ms}$ fixation cross, the

\footnotetext{
1 The AoA provided by Bates et al., (2003) were taken for the words for which Snodgrass and Vanderwart did not provide an AoA.

2 WordGen did not provide the log-transormed lexical frequency for 7 out of the 200 words.
} 
words were displayed for $2000 \mathrm{~ms}$ and followed by a jittered time interval of 1500-2500 ms. The total response time was 3500-4500 ms long. The four word lists used (Session 1 L1-L2, Session 1 L2-L1, Session 2 L1-L2, Session 2 L2-L1) were composed by nouns that were matched for number of letters, number of neighbors, number of spoken syllables and log frequency per million.

\section{Flanker Task}

As nonverbal executive control task, we used the Flanker Task originally designed by Eriksen and Eriksen (1974). In this task, five black symbols (arrows or squares) appeared in the center of the screen. The participants had to indicate the direction of the middle arrow by pressing a right or left key while ignoring the non-target stimuli flanking the target arrow (i.e., congruently or incongruently pointing arrows, or squares). The task included a total of 150 trials, consisting of 50 congruent trials (middle arrow pointing to the same direction as the flanking arrows), 50 incongruent trials (middle arrow pointing to the opposite direction with respect to the flanking arrows), and 50 neutral trials (middle arrow flanked by squares). Stimuli were presented at the center of the screen and displayed in black font color on a light-grey background. They were displayed for $600 \mathrm{~ms}$ and followed by a $850-1850 \mathrm{~ms}$ ISI. The different stimulus conditions (congruent, incongruent, neutral) were presented randomly.

\section{Electrophysiological Recording and Data Preprocessing}

A 64-channel EEG (Biosemi ActiveTwo system, Amsterdam, Netherlands) was recorded at a sampling rate of $1024 \mathrm{~Hz}$. As a quality check for the EEG-signal, we used electrode-offset values (i.e. running average of voltage at each electrode relative to the common mode voltage of the system) of $\pm 30 \mathrm{mV}$. Using Vision Analyzer software (Brain Products $\mathrm{GmbH}$ ), the data was digitally filtered (lowpass: $40 \mathrm{~Hz}$, highpass: $1 \mathrm{~Hz}$, Notch $50 \mathrm{~Hz}$ ) and bad channels were interpolated (range 1-6 electrodes). For each subject, the same channels were interpolated across the four conditions of each task, i.e. Picture Naming: cTBS L1, cTBS L2, Sham L1, Sham L2; Translation: cTBS L1-L2, cTBS L2-L1, Sham L1-L2, Sham L2-L1; Flanker: cTBS congruent, cTBS incongruent, Sham congruent, Sham incongruent. Trials with artifacts exceeding $+-80 \mu \mathrm{V}$ maximal or minimal value within a trial in any channel were automatically rejected. Trials for which participants gave correct responses and which did not contain artifacts exceeding $+-80 \mu \mathrm{V}$ were epoched $-100-700 \mathrm{~ms}$ following stimulus onset. For the picture naming and word translation tasks, trials for which participants gave correct responses within $700 \mathrm{~ms}$ after stimulus presentation were excluded to avoid motor artifacts.
Averaging was performed separately for each condition. Only data of participants whose accepted trial number was equal or above 20 trials in each of the four conditions of each task were included in the analysis. For the electrical neuroimaging analyses (see section "Electrical neuroimaging analysis"), this led to a final group size of 16 participants for the picture naming task, 17 participants for the translation task and 21 participants for the Flanker task. The final amount of averaged trials for each condition of the three tasks were the following: Picture Naming: cTBS L1 $=40.0$ (SD 7.7), cTBS L2=29.2 (SD 4.9), Sham L1 = 42.3 (SD 6.5), Sham L2 = 28.4 (5.2); Translation: cTBS L1-L2 $=31.4$ (SD 4.7), cTBS L2-L1 = 36.9 (SD 4.5), Sham L1-L2=30.5 (SD 4.5), Sham L2-L1 = 36.6 (SD 4.4); Flanker: cTBS congruent $=43.6(\mathrm{SD} 6.8), \mathrm{cTBS}$ incongruent $=43.2(\mathrm{SD} 4.8)$, Sham congruent $=43.5$ (SD 6.7), Sham incongruent $=42.8$ (SD 5.6). As timing tests revealed a constant stimulus presentation delay of $12 \mathrm{~ms}$, the zero-point was shifted by $12 \mathrm{~ms}$.

\section{Statistical Analysis}

\section{Behavior}

Behavioral response times (RTs) of correct responses were analyzed by means of linear mixed-effects models using the statistical software $\mathrm{R}$ (version 3.3.3; R development Core Team 2007). Mixed-effects models were computed with the lme4 package (Version 1.1-15 [44]) and lmerTest package (version 3.0-1). As standards of best practice in the use of mixed-effects models are still in development, we chose to report our analyses based on Linck and Cunnings (2015), who offer advice for the application of mixed-effects models in language research.

For the Picture Naming and Word translation tasks, the analyses included contrast coded fixed effects for stimulation $(-0.5=$ sham, $0.5=\mathrm{cTBS})$ and language $(-0.5=\mathrm{L} 1$, $0.5=\mathrm{L} 2)$ or translation direction $(-0.5=\mathrm{L} 1-\mathrm{L} 2$, $0.5=\mathrm{L} 2-\mathrm{L} 1)$ respectively, in a $2 \times 2$ factorial design. Random intercepts were included for subjects and items and by-subject random slopes were included for stimulation, language, and their interaction. We used log-transformed RTs since visual inspection of the residual plots revealed heteroscedasticity.

For the Flanker Task, the analyses included the contrast coded fixed effects for stimulation $(-0.5=$ sham, $0.5=\mathrm{cTBS})$ and congruency $(-0.5=$ incongruent, $0.5=$ congruent) in a $2 \times 2$ factorial design. Random intercepts were included for subjects and by-subject random slopes were included for stimulation and congruency. In this case, visual inspection of the residual plots revealed no need to log-transform the reaction time data. 
Table 2 Behavioral results picture naming

\begin{tabular}{|c|c|c|c|c|c|c|}
\hline \multicolumn{7}{|c|}{ Log-transformed RTs } \\
\hline & & & & & \multicolumn{2}{|c|}{ Random effects } \\
\hline & & \multicolumn{3}{|c|}{ Fixed effects } & By subject & By items \\
\hline Parameters & Estimate & SE & $\mathrm{t}$ & $\mathrm{p}$ & SD & SD \\
\hline Intercept & 7.05 & 0.03 & 281 & $<0.001$ & 0.1 & 0.15 \\
\hline Stimulation & 0.03 & 0.01 & 2.33 & 0.03 & 0.04 & - \\
\hline Language & 0.33 & 0.03 & 9.8 & $<0.001$ & 0.1 & - \\
\hline $\begin{array}{c}\text { Stimulation } \times \\
\text { Language }\end{array}$ & -0.01 & 0.02 & -0.6 & 0.56 & 0.07 & - \\
\hline
\end{tabular}

Factors were coded using contrast coding, as follows: Stimulation $(-0.5=$ sham, $0.5=c T B S)$, Language $(-.05=\mathrm{L} 1,0.5=\mathrm{L} 2)$. Model formula for RTs: $R T \sim$ Stimulation *Language $+($ Stimulation *Language । Subject $)+(1 \mid$ Item $)$

\section{Electrical Neuroimaging Analysis}

For the resulting ERPs, we used analyses of global field power (GFP) and topography (Buetler et al. 2014; Grieder et al. 2012; Jost et al. 2014, 2018a; Sallard et al. 2014). Such analyses are reference-independent (Michel et al. 2004) and allow disentangling differences in strength of activation from differences in the configuration of the underlying active brain generators across experimental conditions (Michel and Murray 2012; Murray et al. 2008; e.g. Tzovara et al. 2012). Non-parametric randomization tests on the GFP were computed to assess differences in strength of the electric field at the scalp (Koenig et al. 2011; Koenig and Melie-García 2010; Lehmann and Skrandies 1980). Point-to-point topographic analyses of variance (TANOVA) were applied on the normalized data to assess potential differences in topography. With the p-threshold set to $0.05,5000$ randomization runs were computed on subject-wise averaged epochs (Koenig et al. 2011; Koenig and Melie-García 2009). As multiple time frames were tested and to prevent false positive results (Koenig et al. 2011), we also computed a test of multiple comparisons in order to know how many consecutive significant time frames can be expected at a chance level of 0.05 (Grieder et al. 2012). In the following results section, we thus only report those time frames that passed this test of multiple testing. For the translation task, the GFPanalysis and the point-to-point TANOVA were applied with the within-subject factors translation direction (L1-L2 vs L2-L1) and stimulation (sham vs cTBS). For the picture naming task the analyses were applied with the within-subject factors language (L1 vs L2) and stimulation (sham vs cTBS). For the Flanker task the analyses were applied with the within-subject factors congruency (congruent, incongruent) and stimulation (sham vs cTBS).

To further analyze effects of stimulation, electrical source estimations were calculated using a distributed linear inverse solution based on a local autoregressive average (LAURA) regularization approach (Grave de Peralta Menendez et al.
2001; Grave-de Peralta et al. 2004; Michel et al. 2004; Sallard et al. 2014). The solution space was calculated on a realistic head model including 4928 nodes, selected from a $6.7 \mathrm{~mm} \times 6.7 \mathrm{~mm} \times 6.7 \mathrm{~mm}$ grid of voxels equally distributed within the gray matter of the average brain of the Montreal Neurological Institute (MNI). The results of the above-mentioned topographic analyses defined the time period across which intracranial sources were estimated. ERPs for each participant and condition were time-averaged over the period showing a significant topographic difference for the effects of stimulation. Then, intracranial sources were estimated for the resulting one time-sample ERP for each participant and condition and statistically compared at each solution point. In order to control for multiple comparisons, only solutions with a minimal cluster size of 20 points at $\mathrm{p}<0.05$ were retained. For the resulting regions of interest (ROIs), T-values were extracted and analyzed, which then allowed drawing conclusions on the direction of the effects, namely whether the solution points found were more or less activated in the conditions of interest.

As we were interested in the influence of cTBS vs sham on the behavioral and electrophysiological responses, we consider in the following only the effects of stimulation and their interaction terms.

\section{Results}

\section{Behavioral Results}

An effect of stimulation (estimate $=0.03, p=0.03$ ) was found in the Picture Naming task, showing slower responses after cTBS (mean $=1219 \mathrm{~ms}, \mathrm{SD}=241 \mathrm{~ms}$ ) as compared to sham stimulation $($ mean $=1178 \mathrm{~ms}, \mathrm{SD}=239 \mathrm{~ms})$. No interaction between stimulation and language was found. For the Word Translation and Flanker task, the effects of stimulation or their interaction terms did not reach significance. Detailed results of the linear mixed-effects models are reported in 
Table 3 Behavioral results word translation

\begin{tabular}{|c|c|c|c|c|c|c|}
\hline \multicolumn{7}{|l|}{ Log-transformed RTs } \\
\hline & & & & & \multicolumn{2}{|c|}{ Random effects } \\
\hline & & \multicolumn{3}{|c|}{ Fixed effects } & By subject & By items \\
\hline Parameters & Estimate & SE & $\mathrm{t}$ & $\mathrm{p}$ & SD & SD \\
\hline Intercept & 7.128 & 0.03 & 269.25 & $<0.001$ & 0.11 & 0.17 \\
\hline Stimulation & -0.002 & 0.02 & -0.084 & 0.934 & 0.08 & - \\
\hline Translation direction & -0.161 & 0.04 & -4.283 & $<0.001$ & 0.12 & - \\
\hline $\begin{array}{l}\text { Stimulation } \times \text { Transla- } \\
\text { tion direction }\end{array}$ & 0.009 & 0.03 & 0.326 & 0.75 & 0.10 & - \\
\hline
\end{tabular}

Factors were coded using contrast coding, as follows: Stimulation $(-0.5=$ sham, $0.5=$ cTBS $)$, translation direction $(-.05=\mathrm{L} 1-\mathrm{L} 2,0.5=\mathrm{L} 2-\mathrm{L} 1)$. Model formula for RTs: $R T \sim$ Stimulation $*$ Translation direction $+($ Stimulation $*$ Translation direction $\mid$ Subject $)+(1 \mid$ Item $)$
Table 4 Behavioral results flanker task

\begin{tabular}{|c|c|c|c|c|c|}
\hline \multicolumn{6}{|c|}{ Reaction times } \\
\hline \multirow[b]{3}{*}{ Parameters } & \multirow{2}{*}{\multicolumn{4}{|c|}{ Fixed effects }} & \multirow{3}{*}{$\begin{array}{l}\text { Random effects } \\
\text { By subject } \\
\text { SD }\end{array}$} \\
\hline & & & & & \\
\hline & Estimate & SE & $\mathrm{t}$ & $\mathrm{p}$ & \\
\hline Intercept & 446.972 & 7.451 & 59.988 & $<0.001$ & 34.59 \\
\hline Stimulation & -2.831 & 4.577 & -0.618 & 0.543 & 19.01 \\
\hline Congruency & -70.928 & 3.344 & -21.209 & $<0.001$ & 12.10 \\
\hline $\begin{array}{l}\text { Stimulation } \\
\times \text { Congru- } \\
\text { ency }\end{array}$ & 3.822 & 5.209 & 0.734 & 0.471 & 14.10 \\
\hline
\end{tabular}

Factors were coded using contrast coding, as follows: Stimulation $(-0.5=$ sham, $0.5=$ cTBS $)$, Congruency $(-.05=$ incongruent, $0.5=$ congruent $)$. Model formula for RTs: RT $\sim$ Stimulation $*$ Congruency $+($ Stimulation $*$ Congruency $\mid$ Subject $)$

Tables 2, 3, 4. See also Fig. 1 for an illustration of the RTs for each subject.

\section{Electrophysiological Results}

\section{Picture Naming}

The GFP analysis revealed a main effect of stimulation from 547-700 ms showing higher GFP for cTBS compared to sham (see Fig. 2). No significant periods of interaction between the factors language and stimulation were found. The topographic analysis (global map dissimilarity) revealed neither a main effect of stimulation nor an interaction between stimulation and language.

\section{Translation}

The GFP as well as the topographic analyses (global map dissimilarity) revealed no significant periods of difference neither for the factor stimulation, nor for the interaction term. See Fig. 3 for superimposed ERP and GFP waveforms.

\section{Flanker Task}

The GFP analysis revealed a main effect of stimulation from 552-596 ms with higher GFP after sham compared to cTBS stimulation. There was no interaction between congruency and stimulation.

The topographic analysis (global map dissimilarity) revealed a main effect of Stimulation from 556-592 ms. Again, no interaction between stimulation and congruency was found.

Thus, the time window found for the main effect of stimulation in the GFP (552-596 ms) analysis occurred at the same time as topographic differences (556-592 ms). GFP in the presence of topographic differences is less sensitive to measure differences in activation strength originating from specific brain regions. Therefore, electrical source estimations were calculated based on the time window indicating topographic differences (556-592 ms). They revealed lower activation after cTBS compared to Sham in the left hemisphere with local maxima in the left anterior cingulum, caudate, frontal superior gyrus, paracentral lobule and middle/superior gyrus. Lower activation after Sham compared to cTBS stimulation was found in the right middle occipital gyrus (see also Fig. 4).

\section{Discussion}

We investigated the effects of an inhibitory cTBS protocol over the left DLPFC on picture naming, word translation, and a non-verbal inhibition task on the behavioral as well as the electrophysiological level in a group of late bilingual healthy participants. On a behavioral level, an effect of stimulation was found for response times in the picture naming task, while stimulation did not modulate word translation 


\section{Picture Naming}
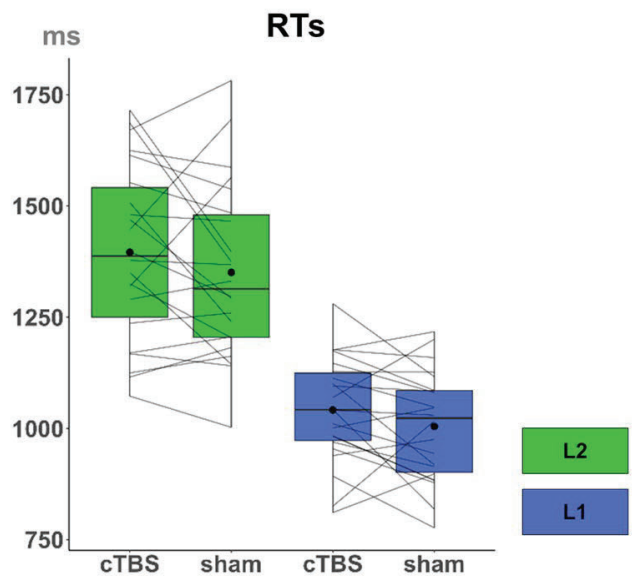

\section{Word Translation}

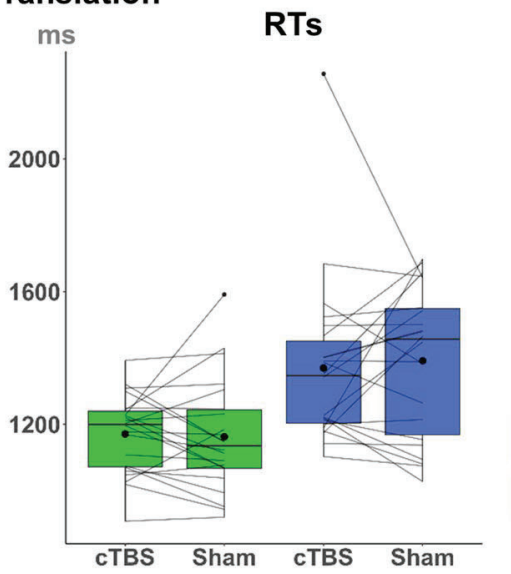

\section{Flanker}

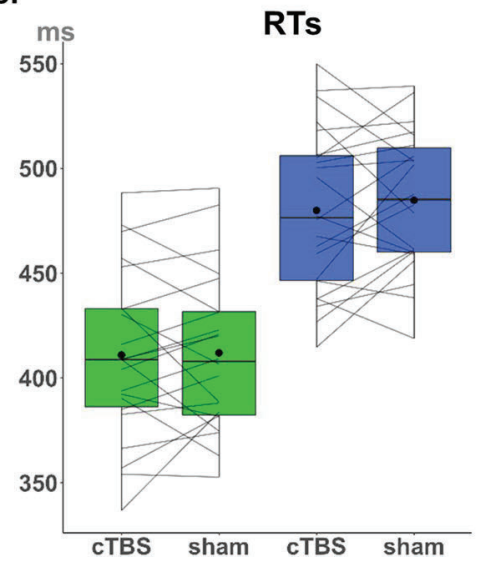

Behavioral results of the picture naming task revealed an effect of stimulation characterized by slower RTs after cTBS compared to Sham. Contrary to our prediction, however, the effect of stimulation was not stronger for naming in L2 than in L1. These results were mirrored on an electrophysiological level, revealing a main effect for stimulation, but no significant interaction. The effect of stimulation was reflected in a stronger activation after cTBS compared to Sham during a late time window from 547-700 ms post-stimulus onset. Differences in similar late time-windows have previously been associated with differences between fast and slow speakers in picture naming, probably associated to differences in motor control strategies and speech motor planning (Laganaro et al. 2012). Moreover, in the bilingual context, this time window has also been found to be associated with cognitive processes of conflict resolution (Martin et al. 2013; Moreno et al. 2002; Ng et al. 2014; Van Der Meij et al. 2011). These results, showing slower verbal response times and electrophysiological changes in motor and cognitive control processes following cTBS, support the notion of a role of the left DLPFC in language control. Previous studies using tDCS to investigate the role of the DLPFC in picture naming have shown differential results. Some reported modulatory effects on response times (Fertonani et al. 2014, 2010; Jeon and Han 2012) while others revealed no effects of stimulation on a behavioral, but only on a neurophysiological level (Radman et al. 2018a; Wirth et al. 2011a). With TMS, we used a stimulation approach that is more focal in targeting specific cortical areas compared to tDCS (Brunoni and Vanderhasselt 2014; Miniussi et al. 2008) and which might have led to a more focal decrease of activity in the DLPFC, therefore expanding the knowledge in this field of research. Interestingly, the effects of stimulation at 547-700 ms post-stimulus onset were independent of the language in use. Similar results have recently been found by Pestalozzi et al. (2020), reporting neurophysiological effects from $533 \mathrm{~ms}$ onwards in both L1 and L2 after applying cTBS over the left DLPFC in a language switching study. Taking into account the meta-analysis on spatio-temporal correlates of language word generation by Indefrey and Levelt (2004, 2011), the effect of stimulation found in the current study occurs in a time range that is associated with the initiation of the articulation of the word, while processes related to lexical and phonological code retrieval as well as syllabification and phonetic encoding are already completed. Moreover, processes of language selection typically start earlier (Khateb et al. 2007). The effects of stimulation thus seem to represent a change in late top-down cognitive and motor control processes, rather than processes related to lexical selection or language switching.

For the translation task, behavioral as well as electrophysiological results did not reveal any effects of stimulation and no interaction. These results question the involvement 


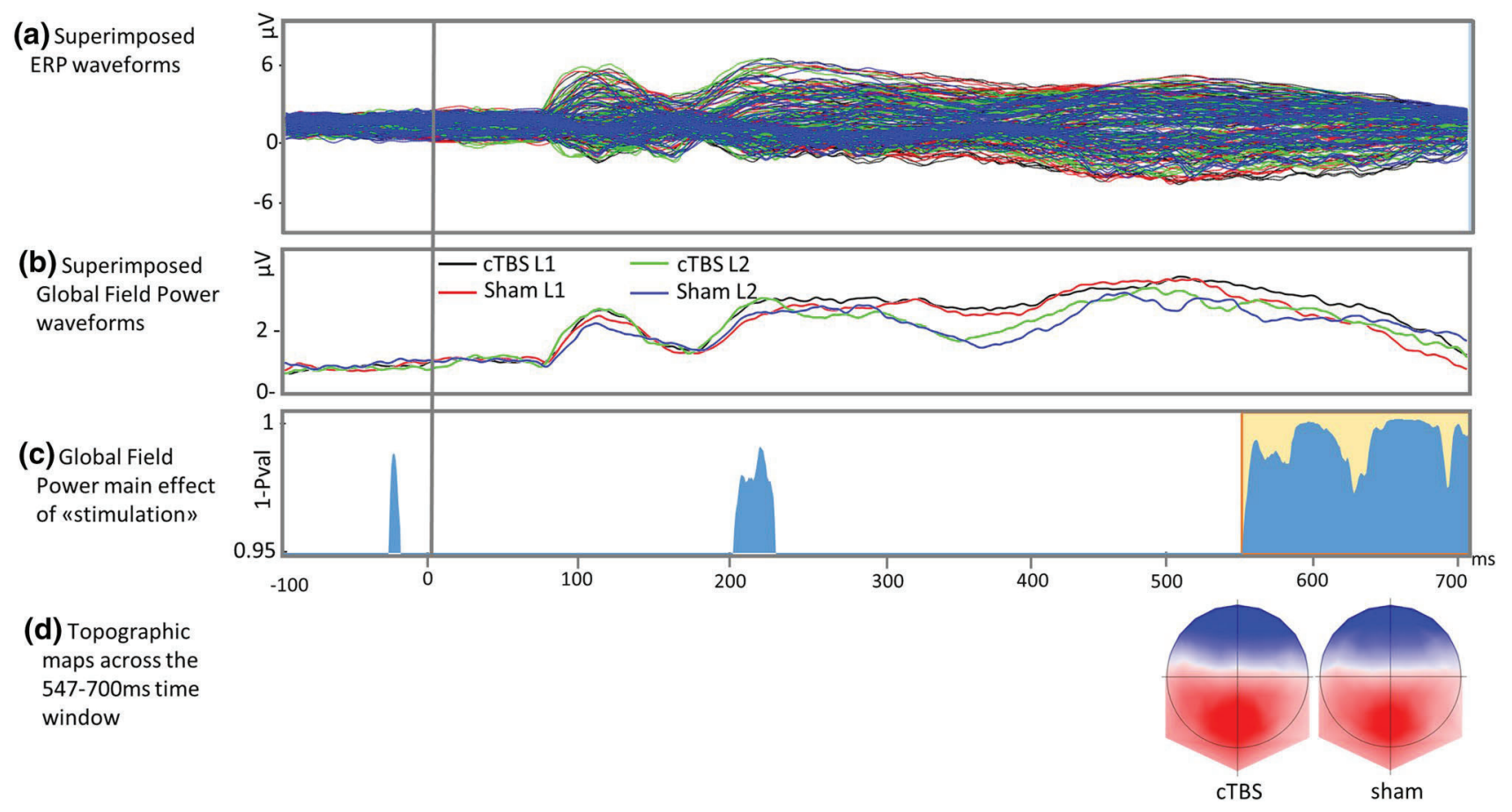

Fig. 2 ERP analyses for Picture Naming. a Superimposed ERP waveforms across all 64 electrodes. b Superimposed Global Field Power waveforms for the four conditions of interest. c Global field power

for the main effect of stimulation $(\mathrm{p}<0.05,>37$ consecutive time frames). d Topographic maps for sham and cTBS across the 547$700 \mathrm{~ms}$ window (colour figure online)
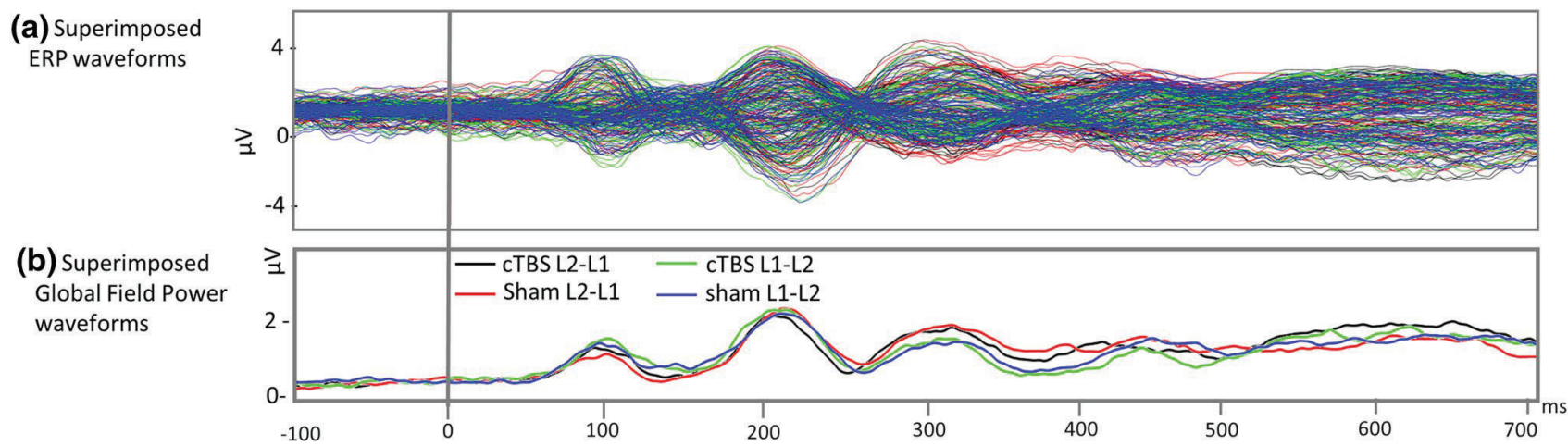

Fig. 3 ERP analyses for forward (L1-L2) and backward (L2-L1) Word Translation. a Superimposed ERP waveforms across all 64 electrodes. b Superimposed Global Field Power waveforms for the four conditions of interest (colour figure online)

of the left DLPFC in word translation. Whereas Klein et al. ((Klein et al. 1995) found an involvement of the DLPFC in forward and backward translation, this finding could not be replicated in later studies (Jost et al. 2018; Price et al. 1999). According to our previous study (Jost et al. 2018), word translation involves voluntary reorientation of attention and response preparation as well as later activation of a global control network, which might depend on other brain areas than the DLPFC, such as the superior temporal gyrus and inferior parietal lobe. Hervais-Adelman et al. (2011), on the other hand, proposed that the control of output in translation tasks is mainly modulated by the basal ganglia. Translation is most likely a more complex task than picture naming, involving a large network for the fine-tuning of the relative activations of the two languages, which relativizes the specific contribution of the left DLPFC.

In the flanker task, we found no behavioral effect, but stimulation affected brain activity at $556-592 \mathrm{~ms}$ poststimulus onset. Source estimations showed a decrease of activity in left cognitive control areas following cTBS as compared to sham stimulation during this time-window. Previous studies, however, showed that reaction times in 


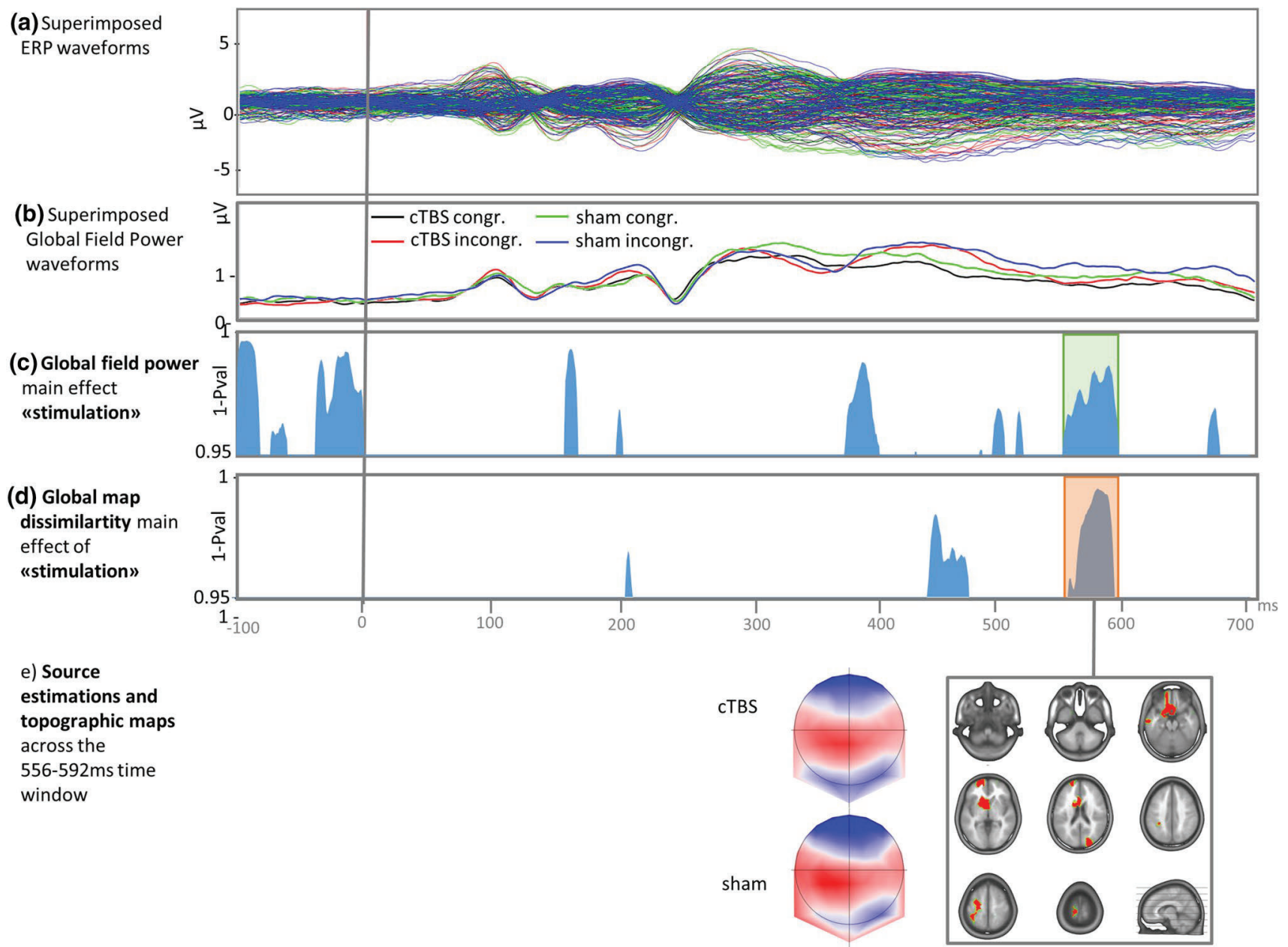

Fig. 4 ERP analyses for the Flanker task. a Superimposed ERP waveforms across all 64 electrodes. b Superimposed Global Field Power waveforms for the four conditions of interest. c Global field power for the main effect of stimulation $(\mathrm{p}<0.05,>43$ consecutive time frames) d Global map dissimilarity of the main effect of stimulation

the nonverbal Flanker task might depend more strongly on right hemispheric areas (Gbadeyan et al. 2016; Luks et al. 2010). As such, it is plausible that a decrease in activity in left hemispheric regions did not affect the behavioral outcome of this task. A methodological limitation that should be noted is that, in contrast to the language tasks, trials with RTs $<700 \mathrm{~ms}$ were not excluded from the ERPs for the flanker task. Overall, the RT's were much faster in the flanker task than in the language tasks, which is why we would have needed to exclude too many trials from the ERPs. It could be argued that only the first $300 \mathrm{~ms}$ of the ERP should be interpreted as the later components could be influenced by the motor response. However, we believe that the ERP results of the flanker task can nevertheless be considered as valid, because: (1) the effect found from 556$592 \mathrm{~ms}$ was an effect of stimulation while the behavioral results showed an effect of congruency, with similar RTs

( $\mathrm{p}<0.05,>36$ consecutive time frames) identifying topographic differences between sham and cTBS stimulation from 556-592 ms. eSource estimations for the significant time window revealing a main effect of stimulation as well as topographic maps for sham and cTBS across the 556-592 ms window (colour figure online)

between the sham and cTBS conditions; (2) in the source analysis, the motor cortex was not differentially activated between sham and cTBS conditions. As such, even though it cannot be completely excluded, it is unlikely that the main effects of stimulation found in the electrophysiological results can be ascribed to the motor responses that fall into the corresponding ERP epoch.

Despite overall significant behavioral effects of stimulation in the picture naming task, it should be taken into account that the inter-individual variability of the behavioral results was high, with only 13 out of 22 participants showing a decrease of RT following cTBS for naming in L1 and 14 out of 22 showing a decrease of RT following cTBS for naming in L2. Moreover, it should be noted that intra-individual variability was also high, reflected in a lack of a response pattern across the three tasks within participants. Such inter and intra-individual variability is a common phenomen in 
brain stimulation studies (Hordacre et al. 2017; Nicolo et al. 2015; Rocchi et al. 2018) and surely highlights that conclusions should be drawn carefully.

Considering the effects of cTBS on subsequent EEG in the present study, we found different effects for each task, with a time window showing larger GFP after cTBS compared to sham in the picture naming task, smaller activation in specific brain areas for the flanker task, and no effects for the translation task. Based on the theoretical notion that cTBS reduces cortical excitability, one might have expected a decrease in GFP rather than an increase in the picture naming task, especially as the RTs for the behavioral results became slower and the EEG results for the flanker task showed decreased activation. However, previous studies investigating the effects of cTBS on electrophysiological measures vary extensively in terms of methodology and results. One interesting study conducted by Rocchi et al. (2018) investigated resting EEG power, local mean field power, TMS-related spectral perturbation and inter-trial phase clustering following cTBS over the motor cortex. Results showed power decreases in resting state EEG in the delta band, while the other electrophysiological measures showed power decrease in the theta band. In contrast, the study conducted by Vernet et al. (2013), also assessing resting EEG and TMS-induced EEG evoked potentials following cTBS over the motor cortex, revealed increased power in the theta band, but decreased power in the beta band, paralleled by a decrease in motor evoked potentials (MEP) size. Furthermore, single-pulse TMS-induced power in the theta and alpha bands was decreased. Another study assessing TMS-evoked potentials (amongst others) in response to cTBS applied over the DLPFC showed a decrease in TMSevoked theta oscillations (Chung et al. 2017). While all these studies considered the effects of cTBS on different frequency bands independently of a cognitive task, Lowe et al. (2018) investigated the effects of cTBS over the DLPFC on ERP amplitudes in response to high/low calorie food stimuli and on a flanker task (an approach methodologically closer to the present study). They found increased P3a amplitudes to high calorie food stimuli and an increase in N2 amplitude to incongruent flanker trials. A study with aphasic patients suggests that inhibitory low-frequency rTMS (inhibitory, but not cTBS) can increase N400 responses (Barwood et al. 2011). In their review of combined TMS-EEG studies, Thut and Pascual-Leone (2010) suggested that larger EEG and evoked potential amplitudes might not be the result of primarily facilitative aftereffects, but could reflect a secondary mechanism compensating for initial inhibition. Similarly, they argued that it can not be excluded that reduced amplitudes could reflect a secondary, potentially protective, mechanisms against initial facilitation. This explanation of compensatory mechanisms is supported by studies reporting both facilitative and suppressive effects within the same experiment on different EEG and evoked potential measures (e.g. Restuccia et al. 2007; Thut et al. 2003).

Another point that could account for some of the discrepancies is related to the DLPFC himself, which has been shown to have a very complex projection mix (Medalla et al. 2007). In this animal study, Medalla et al. (2007) showed that prefrontal pathways innervate spines of excitatory neurons and also synapse with inhibitory neurons in the temporal auditory association cortex. As such, it could be that, depending on the exact location of stimulation within the DLPFC, the effects vary according to which projections were stimulated.

From a methodological perspective, some studies assessed resting state EEG while others TMS-evoked ERPs, some focused on frequency bands and others on ERP amplitudes. Moreover, the majority of analyses applied in the above mentioned studies were conducted with averaged values across clusters of electrodes surrounding the stimulation site (e.g. Barwood et al. 2011; Chung et al. 2017; Lowe et al. 2018; Rocchi et al. 2018). In the present study, we focused on the global ERP measures GFP and topography (and source estimations) considering all recording electrodes. As such, a direct comparison between our study and previous ones is somewhat difficult.

We can summarize that previous studies reveal an inhomogeneous picture, which is also reflected in the present study, and further research is needed to clarify whether cortical excitability is indeed reduced by cTBS, or if the different effects found on the electrophysiological level might depend on: (a) the state of neuronal activation in the targeted brain region at the time of stimulation, as already suggested by Silvanto and Pascual-Leone (2008); (b) the components of the evoked responses, as suggested by Vernet et al. (2013); (c) compensatory mechanisms, as suggested by Thut and Pascual-Leone (2010); and/or (d) the specific projections stimulated by cTBS, especially when it comes to such complex areas as the DLPFC (Medalla et al. 2007).

To conclude, our expectations on the effects of inhibitory cTBS over the left DLPFC were partially confirmed: RTs were longer after cTBS compared to Sham in the picture naming task, while behavioral responses were not affected for word translation. Contrary to our prediction, there was no stronger inhibitory effect on tasks requiring a higher cognitive demand (naming in L2 and L1-L2 translation). Importantly, these behavioral results were mirrored on the electrophysiological level revealing an effect of stimulation only for picture naming. For the non-verbal inhibition task, we found effects of stimulation on the electrophysiological level, but these effects were supposedly not strong enough to affect behavioral responses, or the task was not enough dependent on the left-hemisphere.

Taking up the aim of the study, we conclude that the DLPFC plays a role in top-down cognitive control in 
language, but that this role is not necessarily increased with higher cognitive demand. The DLPFC rather seems to be same as relevant to the dominant as to the non-dominant language, likely due to its late role in processing.

Acknowledgements This work was supported by the Swiss National Science Foundation (No. 325130_156937). We would also like to thank PD Dr. Lucas Spierer for his valuable input throughout the entire study.

\section{Compliance with Ethical Standards}

Conflicts of interest The authors declare that they have no conflict of interest.

\section{References}

Abutalebi DJ, Green DW (2008) Control mechanisms in bilingual language production: neural evidence from language switching studies. Lang Cogn Process 23:557-582. https://doi. org/10.1080/01690960801920602

Abutalebi J, Green D (2007) Bilingual language production: the neurocognition of language representation and control. J Neurolinguistics 20:242-275. https://doi.org/10.1016/j.jneuroling .2006 .10 .003

Alario FX, Ferrand L (1999) A set of 400 pictures standardized for French: norms for name agreement, image agreement, familiarity, visual complexity, image variability, and age of acquisition. Behav Res Methods Instrum Comput J Psychon Soc Inc 31:531-552.

Alario FX, Ferrand L, Laganaro M, New B, Frauenfelder UH, Segui J (2004) Predictors of picture naming speed. Behav Res Methods Instrum Comput 36(1):140-155. https://doi.org/10.3758/bf031 95559.

Badre D, Wagner AD (2004) Selection, integration, and conflict monitoring. Neuron 41:473-487. https://doi.org/10.1016/S0896 $-6273(03) 00851-1$

Barwood CHS, Murdoch BE, Whelan B-M, Lloyd D, Riek S, O'Sullivan JD, Coulthard A, Wong A (2011) Modulation of N400 in chronic non-fluent aphasia using low frequency repetitive transcranial magnetic stimulation (rTMS). Brain Lang 116:125-135. https://doi.org/10.1016/j.bandl.2010.07.004

Bates E, D'Amico S, Jacobsen T, et al (2003) Timed picture naming in seven languages. Psychonomic Bulletin \& Review 10:344-380. https://doi.org/10.3758/BF03196494

Bloem I, Heij La (2003) Semantic facilitation and semantic interference in word translation: implications for models of lexical access in language production. J Mem Lang 48:468-488. https://doi. org/10.1016/S0749-596X(02)00503-X

Bohlhalter S, Vanbellingen T, Bertschi M, Wurtz P, Cazzoli D, Nyffeler T, Hess CW, Müri R (2011) Interference with gesture production by theta burst stimulation over left inferior frontal cortex. Clin Neurophysiol 122:1197-1202. https://doi.org/10.1016/j.clinp h.2010.11.008

Bolton DAE, Staines WR (2011) Transient inhibition of the dorsolateral prefrontal cortex disrupts attention-based modulation of tactile stimuli at early stages of somatosensory processing. Neuropsychologia 49:1928-1937. https://doi.org/10.1016/j.neuro psychologia.2011.03.020

Brunoni AR, Vanderhasselt M-A (2014) Working memory improvement with non-invasive brain stimulation of the dorsolateral prefrontal cortex: a systematic review and meta-analysis. Brain Cogn 86:1-9. https://doi.org/10.1016/j.bandc.2014.01.008
Buetler KA, de León Rodríguez D, Laganaro M, Müri R, Nyffeler T, Spierer L, Annoni J-M (2015) Balanced bilinguals favor lexical processing in their opaque language and conversion system in their shallow language. Brain Lang 150:166-176. https://doi. org/10.1016/j.bandl.2015.10.001

Buetler KA, de León Rodríguez D, Laganaro M, Müri R, Spierer L, Annoni J-M (2014) Language context modulates reading route: an electrical neuroimaging study. Front Hum Neurosci. https:// doi.org/10.3389/fnhum.2014.00083

Cappa SF, Sandrini M, Rossini PM, Sosta K, Miniussi C (2002) The role of the left frontal lobe in action naming: rTMS evidence. Neurology 59:720-723. https://doi.org/10.1212/WNL.59.5.720

Cazzoli D, Chechlacz M (2017) A matter of hand: Causal links between hand dominance, structural organization of frontoparietal attention networks, and variability in behavioural responses to transcranial magnetic stimulation. Cortex 86:230246. https://doi.org/10.1016/j.cortex.2016.06.015

Cazzoli D, Rosenthal CR, Kennard C, Zito GA, Hopfner S, Müri RM, Nyffeler T (2015) Theta burst stimulation improves overt visual search in spatial neglect independently of attentional load. Cortex 73:317-329. https://doi.org/10.1016/j.corte x.2015.09.009

Chung SW, Lewis BP, Rogasch NC, Saeki T, Thomson RH, Hoy KE, Bailey NW, Fitzgerald PB (2017) Demonstration of short-term plasticity in the dorsolateral prefrontal cortex with theta burst stimulation: a TMS-EEG study. Clin Neurophysiol 128:11171126. https://doi.org/10.1016/j.clinph.2017.04.005

Cotelli M, Calabria M, Manenti R, Rosini S, Zanetti O, Cappa SF, Miniussi C (2011) Improved language performance in Alzheimer disease following brain stimulation. J Neurol Neurosurg Psychiatry 82:794-797. https://doi.org/10.1136/jnnp.2009.197848

Cycowicz YM, Friedman D, Rothstein M, Snodgrass JG (1997) Picture naming by young children: norms for name agreement, familiarity, and visual complexity. J Exp Child Psychol 65:171-237. https://doi.org/10.1006/jecp.1996.2356

De Pretto M, Rochat L, Spierer L (2017) Spatiotemporal brain dynamics supporting the immediate automatization of inhibitory control by implementation intentions. Sci Rep 7:10821. https://doi. org/10.1038/s41598-017-10832-x

Dijkstra T, van Heuven WJB (2002) The architecture of the bilingual word recognition system: from identification to decision. Biling Lang Cogn. https://doi.org/10.1017/S1366728902003012

Duyck W, Desmet T, Verbeke LPC, Brysbaert M (2004) WordGen: a tool for word selection and nonword generation in Dutch, English, German, and French. Behav Res Methods Instrum Comput 36:488-499. https://doi.org/10.3758/BF03195595

Eriksen BA, Eriksen CW (1974) Effects of noise letters upon the identification of a target letter in a nonsearch task. Percept Psychophys 16:143-149. https://doi.org/10.3758/BF03203267

Fertonani A, Brambilla M, Cotelli M, Miniussi C (2014) The timing of cognitive plasticity in physiological aging: a tDCS study of naming. Front Aging Neurosci. https://doi.org/10.3389/fnagi .2014 .00131

Fertonani A, Rosini S, Cotelli M, Rossini PM, Miniussi C (2010) Naming facilitation induced by transcranial direct current stimulation. Behav Brain Res 208:311-318. https://doi.org/10.1016/j. bbr.2009.10.030

Francis WS, Augustini BK, Sáenz SP (2003) Repetition priming in picture naming and translation depends on shared processes and their difficulty: evidence from spanish-english Bilinguals. J Exp Psychol Learn Mem Cogn 29:1283-1297. https://doi. org/10.1037/0278-7393.29.6.1283

Francis WS, Gallard SLK (2005) Concept mediation in trilingual translation: evidence from response time and repetition priming patterns. Psychon Bull Rev 12:1082-1088. https://doi.org/10.3758/ BF03206447 
Gbadeyan O, McMahon K, Steinhauser M, Meinzer M (2016) Stimulation of dorsolateral prefrontal cortex enhances adaptive cognitive control: a high-definition transcranial direct current stimulation study. J Neurosci Off J Soc Neurosci 36:12530-12536. https:// doi.org/10.1523/JNEUROSCI.2450-16.2016

Goldsworthy MR, Pitcher JB, Ridding MC (2012) A comparison of two different continuous theta burst stimulation paradigms applied to the human primary motor cortex. Clin Neurophysiol 123:22562263. https://doi.org/10.1016/j.clinph.2012.05.001

de Peralta G, Menendez R, Gonzalez Andino S, Lantz G, Michel CM, Landis T (2001) Noninvasive localization of electromagnetic epileptic activity. I. Method Descr Simul Brain Topogr 14:131-137

Grave-de Peralta R, González-Andino S, Gómez-González CM (2004) The biophysical foundations of the localisation of encephalogram generators in the brain. The application of a distributiontype model to the localisation of epileptic foci. Rev Neurol 39:748-756

Green DW (1998) Mental control of the bilingual lexico-semantic system. Biling Lang Cogn 1:67. https://doi.org/10.1017/S1366 728998000133

Green DW, Abutalebi J (2013) Language control in bilinguals: the adaptive control hypothesis. J Cogn Psychol 25:515-530. https ://doi.org/10.1080/20445911.2013.796377

Grieder M, Crinelli RM, Koenig T, Wahlund L-O, Dierks T, Wirth M (2012) Electrophysiological and behavioral correlates of stable automatic semantic retrieval in aging. Neuropsychologia 50:160 171. https://doi.org/10.1016/j.neuropsychologia.2011.11.014

Grossheinrich N, Rau A, Pogarell O, Hennig-Fast K, Reinl M, Karch S, Dieler A, Leicht G, Mulert C, Sterr A, Padberg F (2009) Theta burst stimulation of the prefrontal cortex: safety and impact on cognition, mood, and resting electroencephalogram. Biol Psychiatry 65:778-784. https://doi.org/10.1016/j.biopsych.2008.10.029

Hart H, Radua J, Nakao T, Mataix-Cols D, Rubia K (2013) Meta-analysis of functional magnetic resonance imaging studies of inhibition and attention in attention-deficit/hyperactivity disorder: exploring task-specific, stimulant medication, and age effects. JAMA Psychiatry 70:185. https://doi.org/10.1001/jamapsychi atry.2013.277

Hellriegel H, Schulz EM, Siebner HR, Deuschl G, Raethjen JH (2012) Continuous theta-burst stimulation of the primary motor cortex in essential tremor. Clin Neurophysiol 123:1010-1015. https:// doi.org/10.1016/j.clinph.2011.08.033

Hernandez AE, Dapretto M, Mazziotta J, Bookheimer S (2001) Language switching and language representation in Spanish-English bilinguals: an fMRI study. NeuroImage 14:510-520. https://doi. org/10.1006/nimg.2001.0810

Hernandez AE, Martinez A, Kohnert K (2000) In Search of the language switch: an fmri study of picture naming in spanish-english bilinguals. Brain Lang 73:421-431. https://doi.org/10.1006/ brln.1999.2278

Hervais-Adelman AG, Moser-Mercer B, Golestani N (2011) Executive control of language in the bilingual brain: integrating the evidence from neuroimaging to neuropsychology. Front Psychol 2:234. https://doi.org/10.3389/fpsyg.2011.00234

Herwig U, Satrapi P, Schönfeldt-Lecuona C (2003) Using the International 10-20 EEG System for Positioning of Transcranial Magnetic Stimulation. Brain Topogr 16:95-99. https://doi. org/10.1023/B:BRAT.0000006333.93597.9d

Holtzheimer P, Fawaz W, Wilson C, Avery D (2005) Repetitive transcranial magnetic stimulation may induce language switching in bilingual patients. Brain Lang 94:274-277. https://doi. org/10.1016/j.bandl.2005.01.003

Hopfner S, Cazzoli D, Müri RM, Nef T, Mosimann UP, Bohlhalter S, Vanbellingen T, Nyffeler T (2015) Enhancing treatment effects by combining continuous theta burst stimulation with smooth pursuit training. Neuropsychologia 74:145-151. https://doi. org/10.1016/j.neuropsychologia.2014.10.018

Hordacre B, Goldsworthy MR, Vallence A-M, Darvishi S, Moezzi B, Hamada M, Rothwell JC, Ridding MC (2017) Variability in neural excitability and plasticity induction in the human cortex: a brain stimulation study. Brain Stimulat 10:588-595. https://doi. org/10.1016/j.brs.2016.12.001

Huang Y-Z, Edwards MJ, Rounis E, Bhatia KP, Rothwell JC (2005) Theta burst stimulation of the human motor cortex. Neuron 45:201-206. https://doi.org/10.1016/j.neuron.2004.12.033

Indefrey P (2011) The spatial and temporal signatures of word production components: a critical update. Front Psychol. https://doi. org/10.3389/fpsyg.2011.00255

Indefrey P, Levelt WJM (2004) The spatial and temporal signatures of word production components. Cognition 92:101-144. https://doi. org/10.1016/j.cognition.2002.06.001

Iyer MB, Mattu U, Grafman J, Lomarev M, Sato S, Wassermann EM (2005) Safety and cognitive effect of frontal DC brain polarization in healthy individuals. Neurology 64:872-875. https://doi. org/10.1212/01.WNL.0000152986.07469.E9

Jeon SY, Han SJ (2012) Improvement of the working memory and naming by transcranial direct current stimulation. Ann Rehabil Med 36:585. https://doi.org/10.5535/arm.2012.36.5.585

Jost LB, Eberhard-Moscicka AK, Frisch C, Dellwo V, Maurer U (2014) Integration of spoken and written words in beginning readers: a topographic ERP study. Brain Topogr 27:786-800. https://doi. org/10.1007/s10548-013-0336-4

Jost LB, Radman N, Buetler KA, Annoni J-M (2018) Behavioral and electrophysiological signatures of word translation processes. Neuropsychologia 109:245-254. https://doi.org/10.1016/j.neuro psychologia.2017.12.034

Khateb A, Abutalebi J, Michel CM, Pegna AJ, Lee-Jahnke H, Annoni J-M (2007) Language selection in bilinguals: a spatio-temporal analysis of electric brain activity. Int J Psychophysiol Off J Int Organ Psychophysiol 65:201-213. https://doi.org/10.1016/j.ijpsy cho.2007.04.008

Kindler J, Schumacher R, Cazzoli D, Gutbrod K, Koenig M, Nyffeler T, Dierks T, Müri RM (2012) Theta burst stimulation over the right broca's homologue induces improvement of naming in aphasic patients. Stroke 43:2175-2179. https://doi.org/10.1161/STROK EAHA.111.647503

Klein D, Milner B, Zatorre RJ, Meyer E, Evans AC (1995) The neural substrates underlying word generation: a bilingual functionalimaging study. Proc Natl Acad Sci 92:2899-2903. https://doi. org/10.1073/pnas.92.7.2899

Koenig T, Kottlow M, Stein M, Melie-García L (2011) Ragu: a free tool for the analysis of eeg and meg event-related scalp field data using global randomization statistics. Comput Intell Neurosci 2011:1-14. https://doi.org/10.1155/2011/938925

Koenig T, Melie-García L (2010) A method to determine the presence of averaged event-related fields using randomization tests. Brain Topogr 23:233-242. https://doi.org/10.1007/s10548-010-0142-1

Koenig, T., Melie-García, L., 2009. Statistical analysis of multichannel scalp field data, in: Michel, C.M., Koenig, T., Brandeis, D., Gianotti, L.R.R., Wackermann, J. (Eds.), Electrical Neuroimaging. Cambridge University Press, Cambridge, pp. 169-190.

Kroll JF, Bialystok E (2013) Understanding the consequences of bilingualism for language processing and cognition. J Cogn Psychol 25:497-514. https://doi.org/10.1080/20445911.2013.799170

Kroll JF, Bobb SC, Hoshino N (2014) Two languages in mind: bilingualism as a tool to investigate language, cognition, and the brain. Curr Dir Psychol Sci 23:159-163. https://doi.org/10.1177/09637 21414528511

Kroll JF, Stewart E (1994) Category interference in translation and picture naming: evidence for asymmetric connections between 
bilingual memory representations. J Mem Lang 33:149-174. https://doi.org/10.1006/jmla.1994.1008

Laganaro M, Valente A, Perret C (2012) Time course of word production in fast and slow speakers: a high density ERP topographic study. NeuroImage 59:3881-3888. https://doi.org/10.1016/j. neuroimage.2011.10.082

Laufer B, Nation P (1999) A vocabulary-size test of controlled productive ability. Lang Test 16:33-51. https://doi.org/10.1177/02655 3229901600103

Lefaucheur J-P, André-Obadia N, Antal A, Ayache SS, Baeken C, Benninger DH, Cantello RM, Cincotta M, de Carvalho M, De Ridder D, Devanne H, Di Lazzaro V, Filipović SR, Hummel FC, Jääskeläinen SK, Kimiskidis VK, Koch G, Langguth B, Nyffeler T, Oliviero A, Padberg F, Poulet E, Rossi S, Rossini PM, Rothwell JC, Schönfeldt-Lecuona C, Siebner HR, Slotema CW, Stagg CJ, Valls-Sole J, Ziemann U, Paulus W, Garcia-Larrea L (2014) Evidence-based guidelines on the therapeutic use of repetitive transcranial magnetic stimulation (rTMS). Clin Neurophysiol 125:2150-2206. https://doi.org/10.1016/j.clinph.2014.05.021

Lehmann D, Skrandies W (1980) Reference-free identification of components of checkerboard-evoked multichannel potential fields. Electroencephalogr Clin Neurophysiol 48:609-621. https://doi. org/10.1016/0013-4694(80)90419-8

Linck JA, Cunnings I (2015) The utility and application of mixedeffects models in second language research: mixed-effects models. Lang Learn 65:185-207. https://doi.org/10.1111/lang.12117

Liston C, Matalon S, Hare TA, Davidson MC, Casey BJ (2006) Anterior cingulate and posterior parietal cortices are sensitive to dissociable forms of conflict in a task-switching paradigm. Neuron 50:643-653. https://doi.org/10.1016/j.neuron.2006.04.015

Lowe CJ, Staines WR, Manocchio F, Hall PA (2018) The neurocognitive mechanisms underlying food cravings and snack food consumption. A combined continuous theta burst stimulation (cTBS) and EEG study. NeuroImage 177:45-58. https://doi. org/10.1016/j.neuroimage.2018.05.013

Lubrano V, Prod'homme K, Démonet J-F, Köpke B (2012) Language monitoring in multilingual patients undergoing awake craniotomy: a case study of a German-English-French trilingual patient with a WHO grade II glioma. J Neurol 25:567-578. https://doi. org/10.1016/j.jneuroling.2011.08.002

Luk G, Green DW, Abutalebi J, Grady C (2012) Cognitive control for language switching in bilinguals: a quantitative meta-analysis of functional neuroimaging studies. Lang Cogn Process 27:14791488. https://doi.org/10.1080/01690965.2011.613209

Luks TL, Oliveira M, Possin KL, Bird A, Miller BL, Weiner MW, Kramer JH (2010) Atrophy in two attention networks is associated with performance on a Flanker task in neurodegenerative disease. Neuropsychologia 48:165-170. https://doi.org/10.1016/j. neuropsychologia.2009.09.001

Martin CD, Strijkers K, Santesteban M, Escera C, Hartsuiker RJ, Costa A (2013) The impact of early bilingualism on controlling a language learned late: an ERP study. Front Psychol. https://doi. org/10.3389/fpsyg.2013.00815

Medalla M, Lera P, Feinberg M, Barbas H (2007) Specificity in inhibitory systems associated with prefrontal pathways to temporal cortex in primates. Cereb Cortex 17:i136-i150. https://doi. org/10.1093/cercor/bhm068

Michel CM, Murray MM (2012) Towards the utilization of EEG as a brain imaging tool. NeuroImage 61:371-385. https://doi. org/10.1016/j.neuroimage.2011.12.039

Michel CM, Murray MM, Lantz G, Gonzalez S, Spinelli L, Grave de Peralta R (2004) EEG source imaging. Clin Neurophysiol 115:2195-2222. https://doi.org/10.1016/j.clinph.2004.06.001

Miniussi C, Cappa SF, Cohen LG, Floel A, Fregni F, Nitsche MA, Oliveri M, Pascual-Leone A, Paulus W, Priori A, Walsh V (2008) Efficacy of repetitive transcranial magnetic stimulation/ transcranial direct current stimulation in cognitive neurorehabilitation. Brain Stimulat 1:326-336. https://doi.org/10.1016/j. brs.2008.07.002

Miyake A, Friedman NP, Emerson MJ, Witzki AH, Howerter A, Wager TD (2000) The unity and diversity of executive functions and their contributions to complex "Frontal Lobe" tasks: a latent variable analysis. Cognit Psychol 41:49-100. https://doi.org/10.1006/ cogp. 1999.0734

Moreno EM, Federmeier KD, Kutas M (2002) Switching languages, switching palabras (words): an electrophysiological study of code switching. Brain Lang 80:188-207. https://doi.org/10.1006/ brln.2001.2588

Mouthon M, Khateb A, Lazeyras F, Pegna AJ, Lee-Jahnke H, Lehr C, Annoni J-M (2019) Second-language proficiency modulates the brain language control network in bilingual translators: an eventrelated fMRI study. Biling Lang Cogn. https://doi.org/10.1017/ S1366728918001141

Murray MM, Brunet D, Michel CM (2008) Topographic ERP analyses: a step-by-step tutorial review. Brain Topogr 20:249-264. https:// doi.org/10.1007/s10548-008-0054-5

Nardone R, De Blasi P, Bergmann J, Caleri F, Tezzon F, Ladurner G, Golaszewski S, Trinka E (2011) Theta burst stimulation of dorsolateral prefrontal cortex modulates pathological language switching: a case report. Neurosci Lett 487:378-382. https://doi. org/10.1016/j.neulet.2010.10.060

Ng S, Gonzalez C, Wicha NYY (2014) The fox and the cabra: An ERP analysis of reading code switched nouns and verbs in bilingual short stories. Brain Res 1557:127-140. https://doi.org/10.1016/j. brainres.2014.02.009

Nicolo P, Ptak R, Guggisberg AG (2015) Variability of behavioural responses to transcranial magnetic stimulation: origins and predictors. Neuropsychologia 74:137-144. https://doi.org/10.1016/j. neuropsychologia.2015.01.033

Nozari N (2019) The dual origin of semantic errors in access deficit: activation vs. inhibition deficit. Cogn Neuropsychol 36:31-53. https://doi.org/10.1080/02643294.2019.1587397

Nyffeler T, Cazzoli D, Hess CW, Müri RM (2009) One Session of repeated parietal theta burst stimulation trains induces long-lasting improvement of visual neglect. Stroke 40:2791-2796. https ://doi.org/10.1161/STROKEAHA.109.552323

Nyffeler T, Cazzoli D, Wurtz P, Lüthi M, von Wartburg R, Chaves S, Déruaz A, Hess CW, Müri RM (2008) Neglect-like visual exploration behaviour after theta burst transcranial magnetic stimulation of the right posterior parietal cortex. Eur J Neurosci 27:1809-1813. https://doi.org/10.1111/j.1460-9568.2008.06154 .x

Nyffeler T, Wurtz P, Lüscher H-R, Hess CW, Senn W, Pflugshaupt T, von Wartburg R, Lüthi M, Müri RM (2006) Extending lifetime of plastic changes in the human brain. Eur J Neurosci 24:29612966. https://doi.org/10.1111/j.1460-9568.2006.05154.x

Pestalozzi MI, Annoni J-M, Müri RM, Jost LB (2020) Effects of theta burst stimulation over the dorsolateral prefrontal cortex on language switching-A behavioral and ERP study. Brain Lang 205:104775. https://doi.org/10.1016/j.bandl.2020.104775

Price CJ, Green DW, von Studnitz R (1999) A functional imaging study of translation and language switching. Brain 122:2221-2235. https://doi.org/10.1093/brain/122.12.2221

Radman N, Britz J, Buetler K, Weekes BS, Spierer L, Annoni J-M (2018) Dorsolateral prefrontal transcranial direct current stimulation modulates language processing but does not facilitate overt second language word production. Front Neurosci. https://doi. org/10.3389/fnins.2018.00490

Restuccia D, Ulivelli M, De Capua A, Bartalini S, Rossi S (2007) Modulation of high-frequency $(600 \mathrm{~Hz})$ somatosensory-evoked potentials after rTMS of the primary sensory cortex: modulation of high-frequency somatosensory-evoked potentials. Eur J Neurosci 
26:2349-2358. https://doi.org/10.1111/j.1460-9568.2007.05828 .X

Rocchi L, Ibáñez J, Benussi A, Hannah R, Rawji V, Casula E, Rothwell $\mathrm{J}$ (2018) Variability and predictors of response to continuous theta burst stimulation: a TMS-EEG study. Front Neurosci. https ://doi.org/10.3389/fnins.2018.00400

Roelofs A (2018) A unified computational account of cumulative semantic, semantic blocking, and semantic distractor effects in picture naming. Cognition 172:59-72. https://doi.org/10.1016/j. cognition.2017.12.007

Roelofs A (2008) Attention to spoken word planning: chronometric and neuroimaging evidence. Lang Linguist Compass 2:389-405. https://doi.org/10.1111/j.1749-818X.2008.00060.x

Roelofs A, Piai V (2011) Attention demands of spoken word planning: a review. Front Psychol. https://doi.org/10.3389/fpsyg .2011 .00307

Rossi S, Hallett M, Rossini PM, Pascual-Leone A (2009) Safety, ethical considerations, and application guidelines for the use of transcranial magnetic stimulation in clinical practice and research. Clin Neurophysiol 120:2008-2039. https://doi.org/10.1016/j.clinp h.2009.08.016

Sallard E, Barral J, Chavan CF, Spierer L (2014) Early attentional processes distinguish selective from global motor inhibitory control: an electrical neuroimaging study. NeuroImage 87:183-189. https ://doi.org/10.1016/j.neuroimage.2013.11.002

Schicktanz N, Fastenrath M, Milnik A, Spalek K, Auschra B, Nyffeler T, Papassotiropoulos A, de Quervain DJ-F, Schwegler K (2015) Continuous theta burst stimulation over the left dorsolateral prefrontal cortex decreases medium load working memory performance in healthy humans. PLoS ONE 10:e0120640. https://doi. org/10.1371/journal.pone.0120640

Sholl A, Sankaranarayanan A, Kroll JF (1995) Transfer between picture naming and translation: a test of asymmetries in bilingual memory. Psychol Sci 6:45-49. https://doi. org/10.1111/j.1467-9280.1995.tb00303.x

Sierpowska J, Fernandez-Coello A, Gomez-Andres A, Camins À, Castañer S, Juncadella M, Gabarrós A, Rodríguez-Fornells A (2018) Involvement of the middle frontal gyrus in language switching as revealed by electrical stimulation mapping and functional magnetic resonance imaging in bilingual brain tumor patients. Cortex. J Devoted Study Nerv Syst Behav 99:78-92. https://doi.org/10.1016/j.cortex.2017.10.017

Silvanto J, Pascual-Leone A (2008) State-dependency of transcranial magnetic stimulation. Brain Topogr 21:1-10. https://doi. org/10.1007/s10548-008-0067-0

Snodgrass, J.G., Vanderwart, M., 1980. A standardized set of 260 pictures: norms for name agreement, image agreement, familiarity, and visual complexity. J. Exp. Psychol. [Hum. Learn.] 6, 174-215.

Steel A, Song S, Bageac D, Knutson KM, Keisler A, Saad ZS, Gotts SJ, Wassermann EM, Wilkinson L (2016) Shifts in connectivity during procedural learning after motor cortex stimulation: a combined transcranial magnetic stimulation/functional magnetic resonance imaging study. Cortex 74:134-148. https://doi, org/10.1016/j.cortex.2015.10.004

Thut G, Pascual-Leone A (2010) A review of combined tms-eeg studies to characterize lasting effects of repetitive tms and assess their usefulness in cognitive and clinical neuroscience. Brain Topogr 22:219-232. https://doi.org/10.1007/s10548-009-0115-4

Thut G, Théoret H, Pfennig A, Ives J, Kampmann F, Northoff G, Pascual-Leone A (2003) Differential effects of low-frequency rTMS at the occipital pole on visual-induced alpha desynchronization and visual-evoked potentials. NeuroImage 18:334-347. https:// doi.org/10.1016/S1053-8119(02)00048-4

Tupak SV, Dresler T, Badewien M, Hahn T, Ernst LH, Herrmann MJ, Deckert J, Ehlis A-C, Fallgatter AJ (2013) Inhibitory transcranial magnetic theta burst stimulation attenuates prefrontal cortex oxygenation. Hum Brain Mapp 34:150-157. https://doi.org/10.1002/ hbm. 21421

Tzovara A, Murray MM, Michel CM, De Lucia M (2012) A Tutorial review of electrical neuroimaging from group-average to singletrial event-related potentials. Dev Neuropsychol 37:518-544. https://doi.org/10.1080/87565641.2011.636851

Van Der Meij M, Cuetos F, Carreiras M, Barber HA (2011) Electrophysiological correlates of language switching in second language learners: Electrophysiological correlates of language switching. Psychophysiology 48:44-54. https://doi.org/10.111 1/j.1469-8986.2010.01039.x

Vanbellingen T, Pastore-Wapp M, Kübel S, Nyffeler T, Schüpfer A-C, Kiefer C, Zizlsperger L, Lutz K, Luft AR, Walther S, Bohlhalter S (2020) Interhemispheric facilitation of gesturing: A combined theta burst stimulation and diffusion tensor imaging study. Brain Stimulat 13:457-463. https://doi.org/10.1016/j.brs.2019.12.013

Vernet M, Bashir S, Yoo W-K, Perez JM, Najib U, Pascual-Leone A (2013) Insights on the neural basis of motor plasticity induced by theta burst stimulation from TMS-EEG. Eur J Neurosci 37:598606. https://doi.org/10.1111/ejn.12069

Wirth M, Rahman RA, Kuenecke J, Koenig T, Horn H, Sommer W, Dierks T (2011) Effects of transcranial direct current stimulation (tDCS) on behaviour and electrophysiology of language production. Neuropsychologia 49:3989-3998. https://doi.org/10.1016/j. neuropsychologia.2011.10.015

Ye Z, Zhou X (2009) Executive control in language processing. Neurosci Biobehav Rev 33:1168-1177. https://doi.org/10.1016/j.neubi orev.2009.03.003

Zhang S, Thompson N (2004) DIALANG: A diagnostic language assessment system (review). can. Mod Lang Rev Rev Can Lang Vivantes 61:290-293. https://doi.org/10.1353/cml.2005.0011 